\title{
EFEITOS DA HIPOTERAPIA NO DESENVOLVIMENTO PSICOMOTOR DA CRIANÇA AUTISTA: RELATO DE CASO
}

Mariana Caetano de Castilho, Mariana Santos de Moraes, Vitória de Medeiros Marçal, Deborah Cristina Luiz Fernani, Francis Lopes Pacagnelli, Rosana Vera de Oliveira Schicotti, Sandra Silva Lustosa, José Maria Bertão, Maria Tereza Artero Prado Dantas

Universidade do Oeste Paulista - UNOESTE, Curso de Fisioterapia, Presidente Prudente, SP. E-mail: mariatereza@unoeste.br

\section{RESUMO}

Entende-se que autismo infantil é a auto absorção da criança em seu próprio mundo. A criança autista possui dificuldade em se expressar com o mundo externo e ter contato com outras pessoas. Objetivo deste estudo foi analisar a evolução do desenvolvimento psicomotor de uma criança com autismo após três meses de hipoterapia. $\mathrm{O}$ atendimento foi realizado em centro de reabilitação equestre no interior de São Paulo, com frequência de uma vez por semana e foi obtido melhora dos escores da escala de desenvolvimento do individuo, principalmente nas áreas de motricidade global, equilíbrio e organização espacial e manutenção das áreas selecionadas do Inventário Portage Operacionalizado analisadas.

Palavras-chave: fisioterapia, transtorno autístico, criança, crescimento e desenvolvimento, terapia assistida por cavalos.

\section{EFFECTS OF HIPPOTHERAPY ON PSYCHOMOTOR DEVELOPMENT OF AUTIST CHILD: CASE REPORT}

\section{ABSTRACT}

It is understood that infantile autism is the child's self absorption in their own world. The autistic child has difficulty expressing themselves with the outside world and have contact with other people. This study aimed to analyze the development of psychomotor development of a child with autism after three months of hippotherapy. The service was executed in an equestrian rehabilitation center in the interior of São Paulo, with frequently than once a week and was obtained better scores in the motor development of the individual especially in the areas of global motor skills, balance and spatial organization and maintenance of selected areas Operating Portage Inventory analyzed.

Keywords: physical therapy specialty, autistic disorder, child, growth and development, equine-assisted therapy.

\section{INTRODUÇÃO}

Entende-se que o autismo infantil é a auto absorção da criança em seu próprio mundo. A criança autista possui dificuldade em se expressar com o mundo externo, fato que a prejudica devido à dificuldade de contato com outras pessoas, já que este contato é essencial para o desenvolvimento humano ${ }^{1}$. Os autistas têm características comportamentais particulares, tais como, apresentar atenção em situações que são indiferentes para pessoas típicas, podem se distrair com objetos de utilidades diária e simples, manipulando brinquedos de maneira diferente. Existem dificuldades para se diagnosticar o autismo, pois seus principais prejuízos só se manifestam geralmente após os três anos de idade, portanto as observações e informações dos pais são fundamentais ${ }^{2,3}$.

A manifestação do transtorno varia em cada indivíduo, inclusive as suas características e o seu desenvolvimento. No entanto, não existe cura para indivíduos diagnosticados com autismo, mas existem intervenções que podem melhorar suas habilidades de comunicação, socialização e funções motoras. Dentre estas, a hipoterapia é uma das intervenções que pode colaborar com o desenvolvimento da criança, devido ao uso do 
cavalo como instrumento terapêutico para $o$ estímulo sensorial e motor da criança, também com intuito de ganho de força muscular, melhora no equilíbrio e conscientização corporal ${ }^{4-6}$.

$\mathrm{Na}$ hipoterapia as crianças criam uma afinidade com o cavalo, o que facilita a terapia, principalmente com estas que apresentam dificuldade na socialização, pois estas se sentem seguras e confiantes com a aproximação ao animal, além disso, um fator importante é que os cavalos são não verbais, isso reforça a sensação de liberdade e segurança para criança, sem medo de contestação ou crítica, ${ }^{7,8}$.

As atividades realizadas na hipoterapia buscam estimular: a propriocepção, para que a criança desenvolva a consciência corporal; o tato, que é estimulado pelo toque na pelagem do animal, ao fazer carinho ou escová-lo; a audição, que pode ser aguçada com o ritmo dos cascos ou com a voz do acompanhante; o olfato, que é estimulado pelas informações advindas do próprio cheiro do animal e do ambiente de equitação; o visual pode ser estimulado pela visão expandida de cima do cavalo; além da realização de atividades pedagógicas que promovem melhora da aprendizagem ${ }^{9}$.

Portanto, o objetivo deste estudo foi analisar o desenvolvimento psicomotor de uma criança com autismo após três meses de hipoterapia.

\section{METODOLOGIA}

Este estudo trata-se de um relato de caso, que foi aprovado por comitê de ética em pesquisa, com o CAAE no 46157215.0.0000.5515. A criança G.M.J.A., do sexo masculino, apresentava 10 anos de idade e havia sido encaminhada a um centro de reabilitação equestre no interior de São Paulo, com diagnóstico clínico de Autismo, sendo avaliada pela equipe com uso de instrumentos que classificam o autismo (Childhood Autism Rating Scale - (ARS) ${ }^{10}$ e verificam o desenvolvimento psicomotor da criança (Escala de Desenvolvimento Motor ${ }^{11}$ e Inventario Portage Operacionalizado ${ }^{12}$ ), em seguida recebeu intervenção psicomotora na reabilitação equestre por três meses, sendo reavaliada ao final, com os mesmos intrumentos.

O Childhood Autism Rating Scale (CARS) é um instrumento que classifica o autismo em leve/moderado e grave. Sua escala é constituída por 15 itens, que avaliam o indivíduo em: relações pessoais, imitação, resposta emocional, uso corporal, uso de objetos, resposta a mudanças, resposta visual, resposta auditiva, resposta e uso ao paladar, olfato e tato, medo ou nervosismo, comunicação verbal, nível de atividade, nível e consistência da resposta intelectual e impressões gerais. Com a pontuação do instrumento é possível classificar o indivíduo que não apresenta autismo (com pontuação de 15 a 30 pontos), com autismo leve/moderado (de 30 a 36 pontos) e com autismo grave (de 36 a 60 pontos) ${ }^{10}$.

A Escala de Desenvolvimento Motor (EDM), desenvolvida por Rosa Neto (2002), verifica o desenvolvimento motor em diferentes áreas, como: motricidade fina (movimentos finos com as mãos), motricidade global (movimentos coordenados e amplos), esquema corporal (reconhecimento da imagem do seu corpo no ambiente), equilíbrio (postura estática), organização espacial (reconhecimento do espaço), organização temporal (noção de tempo e linguagem) e lateralidade (preferência no uso de um lado do corpo) ${ }^{11}$.

Para a análise do desenvolvimento motor, a EDM apresenta uma pontuação denominada quociente motor de cada área avaliada, e o resultado final da escala (que engloba todas as áreas) é denominado de quociente motor geral ( $Q M G$ ), o qual é possível classificar o individuo em Muito Superior (com a pontuação de 130 ou mais, com nenhum fator de risco para o desenvolvimento), Superior (com 120 a 129 pontos, com nenhum fator de risco), Normal alto (com 110 a 119 pontos sem nenhum fator de risco), Normal médio (com 90 a 109 pontos com o fator de risco inexistente), Normal baixo (com pontuação de 80 a 89 , com fator de risco leve), Inferior (com 70 a 79 pontos com o fator de risco moderado) e Muito inferior (com pontuação de 69 ou menos com o risco grave para o desenvolvimento). Nesta avaliação o indivíduo que apresentar classificação igual ou inferior a Normal baixo necessita de intervenção.

Também foi utilizado o Inventário Portage Operacionalizado (IPO), um instrumento de avaliação do desenvolvimento psicomotor de crianças do nascimento aos seis anos de idade, nas áreas de estimulação infantil, socialização, cognição, auto cuidados, desenvolvimento motor e linguagem. Este inventário é direcionado aos pais ou responsáveis e seus resultados são apresentados em percentual de acertos ${ }^{12}$. No 
entanto, mesmo que a criança deste estudo apresentava faixa etária superior a de indicação do instrumento, este foi selecionado pois verifica diversos comportamentos, que geralmente apresentam-se comprometidos em indivíduos com autismo. Além de serem utilizadas somente as áreas de socialização, cognição e autocuidados, como forma de complementar a avaliação da criança com a EDM.

A intervenção, na hipoterapia iniciou em Setembro de 2015 e foram realizadas 13 sessões, com média de 50 min pelo turno da manhã, com frequência de uma vez por semana e duração de 3 meses. $O$ ambiente do centro de reabilitação equestre é ao ar livre e constituído de um cerco de $20 \mathrm{~m}$ de largura por $40 \mathrm{~m}$ de cumprimento, com a existência de uma rampa auxiliadora para facilitar a montaria da criança, deixando-o na mesma altura do dorso do animal.

Para o tratamento foram elaborados exercícios que estimulassem cada área do desenvolvimento psicomotor. Foram realizados exercícios que exigissem da motricidade fina, por três series de dez repetições onde a criança precisava pegar as bolinhas e jogar para seu acompanhante. Também foram realizados, com três series de dez repetições e descanso de 60 seg, exercícios de abdução de ombro bilateral com faixa elástica amarela (Carciband), flexão e extensão de tronco com estimulação tátil de acariciar a pelagem do cavalo e depois retornar a posição ereta de tronco. Houve incentivo a criança a segurar as rédeas e deslocamento do tronco ao colocar a bola em suportes de pvc colocados dentro do cerco e na direção do trajeto do cavalo. Sendo que estas atividades estimulam também as áreas de equilíbrio, lateralidade e motricidade global.

No esquema corporal e organização espacial, foram realizados três vezes a atividade de serpenteio com o cavalo entre os suportes de pvc e neste trajeto com o cavalo a passos, intercalou com a atividade da criança colocar o bambolê em si mesma e depois no acompanhante. A organização temporal foi trabalhada com perguntas durante os exercícios, para que a criança criasse respostas temporais, formando frases com as palavras que davam sequencialidade, noção de tempo e horários.

\section{RESULTADOS}

O participante deste estudo apresentou a classificação do CARS com 30 pontos, o que indica autismo leve-moderado. Os achados em relação a comparação entre as pontuações das avaliações do desenvolvimento do indivíduo, antes e após a intervenção da hipoterapia estão apresentados abaixo.

Ao analisar a Figura 1 na primeira avaliação da EDM, foi verificado atraso psicomotor em todas as áreas da escala, sendo que o indivíduo obteve a classificação do quociente motor geral (QMG $=60,5$ pontos) como Muito inferior, com o fator de risco grave para o desenvolvimento. Dentre estas áreas, o equilíbrio (QM3), a organização espacial (QM4) e a organização Temporal (QM6) foram as áreas mais deficitárias.

Após a intervenção da hipoterapia, o indivíduo ainda apresentou atraso psicomotor, porém obteve melhora na área de equilíbrio, organização espacial e motricidade global em comparação a avaliação inicial (Figura1). No entanto, houve regressão na pontuação das áreas de motricidade fina, esquema corporal e organização temporal (Figura 1). Já a classificação do desenvolvimento motor, evoluiu de muito inferior na avaliação, para Inferior na reavaliação com o fator de risco moderado (QMG $=72$ pontos).

A Tabela 1 apresenta os resultados da análise do IPO antes e após a intervenção da hipoterapia, nas áreas selecionadas de socialização, cognição e auto cuidados. Foi verificado na avaliação que a criança apresentou baixos percentuais de acertos, pois devido a sua faixa etária, esta deveria obter $100 \%$ de acertos em todas as áreas. E após a intervenção, somente houve diminuição dos percentuais na área de socialização e cognição, pois na maioria dos itens avaliados houve a manutenção ou melhora destes percentuais, contudo, estes percentuais ainda eram inferiores a idade. 


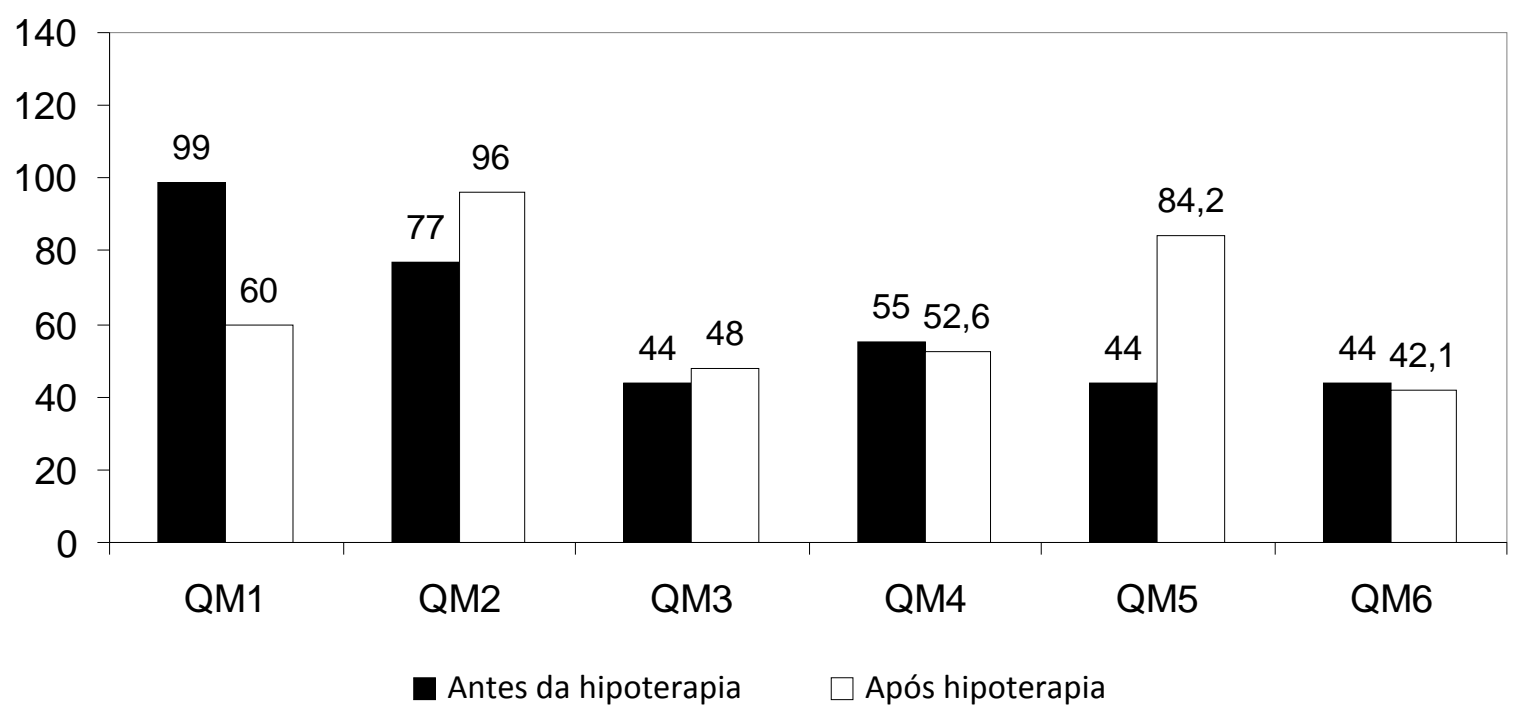

Figura 1. Quocientes motores (em pontos) nas diferentes áreas da Escala de desenvolvimento motor. QM1motricidade fina; QM2- motricidade global; QM3- equilíbrio; QM4- esquema corporal; QM5- organização espacial; QM6- Organização temporal.

Tabela 1. Dados da avaliação do IPO antes e após o período de intervenção na hipnoterapia.

\begin{tabular}{lcccccc}
\hline Idade & \multicolumn{2}{c}{ Socialização (\%) } & \multicolumn{2}{c}{ Cognição (\%) } & \multicolumn{2}{c}{ Auto-cuidados (\%) } \\
\hline (anos) & Antes & Após & Antes & Após & Antes & Após \\
\hline 5 - 6 & 27,3 & 27,3 & 31,8 & 40,9 & 46,6 & 53,0 \\
4 -5 & 33,3 & 55,5 & 59,0 & 59,0 & 47,7 & 52,1 \\
3 - 4 & 33,3 & 41,6 & 95,8 & 54,1 & 33,3 & 33,3 \\
2 - 3 & 77,7 & 77,7 & -- & -- & 81,4 & 81,4 \\
1 - 2 & 60,0 & 66,6 & -- & -- & 91,6 & 91,6 \\
0 -1 & 100 & 96,4 & -- & -- & 100 & 100 \\
\hline
\end{tabular}

\section{DISCUSSÃO}

Na avaliação inicial pela EDM, a criança apresentou a classificação de muito inferior e com o fator de risco grave para o desenvolvimento psicomotor, após a intervenção com a hipoterapia, foi verificada evolução para a classificação inferior e com o fator de risco moderado, devido à melhora obtida pela criança nas áreas de equilíbrio, organização espacial e motricidade global. Porém, foi observado uma regressão nas áreas de motricidade fina, esquema corporal e organização temporal.

No período inicial do tratamento $o$ paciente apresentou-se calmo e obteve adequada adaptação e interação com os cavalos, terapeutas e o ambiente, cooperando nas atividades solicitadas durante a montaria. Assim, acredita-se que o ambiente de tratamento e o animal proporcionam atividades que trabalham o desenvolvimento motor, aspecto emocional e social.

A sessão de hipoterapia teve o objetivo de trabalhar cada área do desenvolvimento psicomotor. Além disso, as atividades desempenhadas associadas ao andar tridimensional do cavalo, desafiavam respostas motoras ao corpo da criança, garantindo bons resultados no desenvolvimento global. Sendo visualizada melhora nas áreas de equilíbrio e motricidade global da criança, como também encontrado no estudo de Barbosa e Munster ${ }^{13}$, que avaliou crianças com Transtorno do Déficit de Atenção com Hiperatividade (TDAH) e 
verificou que após as sessões de hipoterapia houve melhoras destas respectivas áreas. ${ }^{13}$

Foi obtido também melhora na pontuação na organização espacial, além de ser percebido que a criança começou a desenvolver melhor a percepção do ambiente. Esses dados foram ao encontro dos achados no estudo de Brilinger $^{14}$ que também utilizou a hipoterapia como intervenção, porém na Síndrome de Down, obtendo melhoras na área de organização espacial.

Ao começar a frequentar um ambiente com diferentes estímulos, houve interferências no desenvolvimento da área de esquema corporal, a qual é necessária para o reconhecimento do próprio corpo. No estudo de Niehus e Niehus ${ }^{15}$, houve bons resultados após a hipoterapia, na área de esquema corporal com crianças que apresentam TDAH no ambiente escolar. Sabe-se que o passo e o movimento trimendisional do cavalo, há o ganho do realinhamento corporal e consequentemente melhora na percepção e noção corporal ${ }^{15}$.

$\mathrm{Na}$ organização temporal, a criança não evoluiu na pontuação, porém já consegue diferenciar os períodos do dia e seguir diferentes ritmos. Ao comparar estes achados com os do estudo de Prestes et al. ${ }^{16}$, que avaliou dois indivíduos com dificuldades na aprendizagem e obtiveram progresso em todas as áreas psicomotoras, inclusive na organização temporal após a hipoterapia, achado que difere deste estudo.

Para a melhora de motricidade fina é necessário a junção do estímulo tátil e da visão. Mesmo sendo visualizada com destaque na capacidade para desenhos que a criança apresentou, não foi verificado melhora da pontuação na área. A regressão desta pode ser devido a não fixação do olhar da criança quando está interagindo com outra pessoa, dando preferência ao estímulo auditivo da voz do acompanhante, assim o estímulo tátil deixa de ser o foco, fato descrito por Davidse ${ }^{17}$.

Em relação a avaliação com o IPO, os resultados deste estudo demonstraram que a criança apresentou baixos percentuais de acertos, em relação a sua faixa etária, pois esta deveria obter $100 \%$ de acertos nas áreas. No entanto, após a intervenção da hipoterapia a maioria dos itens avaliados por este instrumento, mantive ou melhorou os percentuais de acertos. Somente foi verificada regressão nos percentuais das áreas de socialização e cognição, pois acredita-se que este achado foi devido a mudança de rotina da criança durante o período de intervenção, pela modificação da turma escolar e mudança do professor da criança, o que a afetou emocionalmente. E segundo estudo de Pereira et al. ${ }^{18}$, os autistas tem resistência a mudança de rotina, e para obter bons resultados nestas áreas é preciso de estabilidade emocional para o desenvolvimento de hábitos diários.

A hipoterapia tem se mostrado um método eficaz de tratamento, no entanto, não houve maiores ganhos, provavelmente devido ao curto período do tratamento (três meses), justificado pela intercorrência de adoecimento respiratório da criança durante este período. Mesmo com os resultados notórios, mais estudos na área são necessários para contribuir com o conhecimento do avanço psicomotor destes indivíduos.

Portanto, pode-se concluir que após três meses de hipoterapia houve evolução do desenvolvimento psicomotor do individuo com autismo.

\section{CONFLITO DE INTERESSES}

Os autores declararam não haver qualquer potencial conflito de interesse que possa interferir na imparcialidade deste trabalho cientifico.

\section{REFERÊNCIAS}

1. Stone $\mathrm{Wl}$, Turner L. O impacto do autismo no desenvolvimento infantil. Enciclopédia sobre o Desenvolvimento na Primeira Infância. 2011. [acesso em 2016 jun 08]. Disponível em: http://www.enciclopediacrianca.com/autismo/segundo-especialistas/oimpacto-do-autismo-no-desenvolvimento-infantil

2. Ramos CSA, Salomão NRM. Autismo e síndrome de down: concepções de profissionais de diferentes áreas. Psicol Estudo. 2014;19(1):103-14. DOI: https://doi.org/10.1590/1413-7372189590010

3. Perera $\mathrm{A}$, Cruz $\mathrm{ACB}$, Bonatto $\mathrm{CL}$, Rech $\mathrm{FZ}$, Simonaggio LS, Giacomet D, Schunter RC. Análise do padrão de marcha do espectro autista. II Congresso de Pesquisa e Extensão da FSG. 2014:361-9.

4. Borgesi MBS, Wernecki MJS, Silvall ML, Gan dolfill L, Pratesil R. Efeitos terapêuticos de um simulador de equitação em crianças com paralisia cerebral. Arq Neuro-Psiquiatr. $\quad 2011 ; 69(5): 799-804 . \quad$ DOI: https://doi.org/10.1590/S0004-282X2011000600014 
5. Cirulli F, Borgin M, Francia N, Alleva E. Animalassisted interventions as innovate for mental health. Ann Ist Supper Sanitá. 2011;47(4):341-8. DOI: https://doi.org/10.4415/ANN 110404

6. Lemke D, Rothweell E, Neuromb TM, Swoboda KJ. Perceptions of equine assisted ativities and therapies by parentes and children witch spinal muscular atrophy. Pediatr Phys Ther. 2014;26(2):237-44. DOI: https://doi.org/10.1097/PEP.0000000000000027

7. Granados $A C, A g$ 'is IF. Why children with special needs feel better with hippotherapy sessions: a conceptual review. J Alternat Complement Med. 2011;17(3):191-7.

DOI: https://doi.org/10.1089/acm.2009.0229

8. Soares T, Braga SEM. Relação de terapia de holding com integração sensorial no autismo infantil. Rev Cient Interdiscipl. 2014;1(2):78-159. DOI: https://doi.org/10.17115/2358-8411/v1n2a6

9. Freire HBG, Potsc HRR. O autista na equoterapia: a descoberta do cavalo. UCDB. 2015. [acesso em 2016 jun 8] Disponível em: http://www.universoautista.com.br/autismo/modules $\angle$ news/article.php?storyid $=476$

10. Pereira A, Riesgo RS, Wagner MB. Autismo infantil: tradução e validação da Childhood Autism Rating Scale para uso no Brasil. J Pediatr. 2008;84(6):487-94. DOI: https://doi.org/10.1590/S0021-75572008000700004

11. Rosa Neto F. Manual de avaliação motora. 2.ed. São Paulo: Artmed Editora; 2002. 144p.

12. Willians LCA, Aiello ALR. O inventário Portage operacionalizado. São Paulo: Memnon Edições Cientificas Ltda.; 2002. 299p.

13. Barbosa GO, Munster MA. O efeito de um programa de equoterapia no desenvolvimento psicomotor de crianças com indicativos de transtorno de déficit de atenção e hiperatividade. Rev Bras Educ Esp. 2014;20(1):69-84.

14. Brilinger, CO. A influência da equoterapia no desenvolvimento motor do portador de Síndrome de Down: estudo de um caso. [Monografia]. Bacharelado em Fisioterapia. Universidade do Sul de Santa Catarina. 2005. 110p.

15. Niehues JR, Niehues MR. Equoterapia no tratamento de transtorno de Déficit de Atenção e Hiperatividade (TDAH): implicações pedagógicas. Rev. Neurocienc. 2014;22(1):121-6. DOI: https://doi.org/10.4181/RNC.2014.22.902.6p
16. Prestes DB, Weiss S, Araújo JCO. A equoterapia no desenvolvimento motor e autopercepção de escolares com dificuldade de aprendizagem. Ciên Cognição. 2010;15(3):192-203.

17. Davidse CL. Ouvir para se poder olhar dentro da clínica do autismo. De onde vem a voz que me faz existir? Rev Latinoam Psicopat. 2015;18(4):634-50. DOI: $\quad$ https://doi.org/10.1590/14154714.2015v18n4p634.4

18. Pereira CCV, Borges TAS, Marques RRC. Tratamento e evolução de crianças autistas atendidas em uma associação de João Pessoa. Rev Ciênc Saúde Nova Esperança. 2015;13(1):77-85.

Recebido para publicação em 16/06/2016

Revisado em 05/07/2017

Aceito em 10/05/2018 